\title{
Conventional and targeted therapy in metastatıc colorectal cancer
}

\begin{abstract}
Colorectal cancer is one of the leading causes of cancer related mortalities and its dismal prognosisis is well known. $20 \%$ to $25 \%$ of colorectal cancer patients have advanced stage disease and systemic chemotherapy is the main treatment for these patients for palliation. But there is novel treatment options for metastatic colorectal patients. With these novel options median survivals have prolonged from 12 months to nearly 40 months In this review, we discussed the conventional treatments and new therapy approaches for metastatic colorectal cnacer patients.
\end{abstract}

Keywords: Colorectal cancer, Conventional, Targeted therapies
Volume 9 Issue 6 - 2018

\author{
Ali Murat SEDEF,' Ahmet Taner SÜMBÜL ${ }^{2}$ \\ 'Sanliurfa Training and Research Hospital, Department of \\ Medical Oncology,Turkey \\ ${ }^{2}$ Baskent University Faculty of Medicine, Department of Medical \\ Oncology, Turkey
}

Correspondence: Ahmet Taner Sümbül, Sanliurfa Training and Research Hospital, Department of Medical Oncology, Turkey, Tel 0090 5056166338, Fax 00903223444445 Email drtanarsu@yahoo.com

Received: December 01, 2016 | Published: November 16 2018

\section{Introduction}

Colorectal cancer is the second leading cause of death in the United States among all cancers. 1 Approximately $20 \%$ to $25 \%$ of colorectal cancer patients are diagnosed with liver metastasis at the time of initial diagnosis and a further $40 \%$ to $50 \%$ of patients will develop liver metastasis within 3 years from initial diagnosis. ${ }^{2,3}$ Metastatic colorectal cancer is generally not curable disease. However, a subset of patients with isolated metastases to liver and/or lung are potentially curable with local therapies such as surgery. Treatment is systemic chemotherapy with purpose palliation and control of symptoms, control of tumor growth for patients with non-curable metastatic colorectal cancer. The median survival is 5 to 6 months for metastatic colorectal cancer patients without specific therapy. After fluorouracil plus leucovorin (LV) therapy median survival is approximately 1 year for these patients. The addition of oxaliplatin and irinotecan to the fluorouracil and LV therapy provided an improvement in median survival to nearly 20 months since the year 2000 . Finally new pathways has been defined and new therapies started to use which targeted these new pathways. Overall survival was increased with these novel agents such as bevacisumab, cetuximab, panitumumab, other TKİs and immunotherapies. ${ }^{4}$

\section{Systemic chemotherapy options}

Fluorouracil and leucovorin: Fluorouracil (5-FU) has been wildly used as a primary medication in the treatment of solid tumors including colorectal cancer. Although 5-fluorouracil (5-FU) has been used to treat advanced colorectal cancer for 45 years, the drug has only a modest response rate and impact on survival. Until 2000, standard first-line therapy for metastatic colon cancer was the fluoropyrimidine based therapies. This treatment consisted of fluorouracil (FU) and leucovorin. Fluorouracil is a cytotoxic agent which effects by inhibition of DNA and RNA synthesis. ${ }^{5}$ Leucovorin (LV) is biomodulator and activator for florouracil with inhibition of the enzyme thymidylate synthase and and Leucovorin enhances FU cytotoxicity by interacting with thymidylate synthase. Response rates of this combination are in the range of $15 \%$ to $25 \%$ and compared to $\mathrm{FU}$ alone, $\mathrm{FU} / \mathrm{LV}$ is associated with a twofold higher response rate (21 versus 11 percent) in two metaanalyses, ${ }^{6,7}$ These drugs has been given in various schema and doses such as the Mayo Clinic regimen (425 $\mathrm{mg} / \mathrm{m} 2$ of fluorouracil and $20 \mathrm{mg} / \mathrm{m}^{2}$ for LV on days 1 to 5 every 4 to 5 weeks) and The Roswell Park regimen $(500 \mathrm{mg} / \mathrm{m} 2$ of fluorouracil and $500 \mathrm{mg} / \mathrm{m}^{2}$ of LV administered weekly for 6 out of 8 weeks). ${ }^{8,9}$ Studies comparing different methods of 5-FU administration (bolus injection $\mathrm{v}$ prolonged infusion) have demonstrated improved response rates and decreased toxicity with prolonged infusion, but most have failed to demonstrate statistically significant improvements in survival.

Capecitabine: Capecitabine is an oral fluoropyrimidine agent. It is precursor of fluorouracil and metabolized to active form after enzymatic reactions in the liver. The efficacy of capecitabine is similar to FU/LV combinations. Oral capecitabine monotherapy has been shown to have superior antitumor activity to bolus fluorouracil with leucovorin (Mayo Clinic regimen) in this setting, with higher response rates $(26 \%$ vs. $17 \%, \mathrm{p}<0.0002)$ and at least equivalent TTP and OS in two large randomized studies. ${ }^{10}$ The approved dose is $1250 \mathrm{mg} / \mathrm{m}$ twice daily for 14 of every 21 days. In animal model experiments, a higher therapeutic index (ratio of the 50\% toxic dose and the 50\% effective dose) compared to other fluoropyrimidine agents has been reported, ${ }^{11}$ suggesting that less dosage may still maintain good efficacy with a less toxic profile. Most common side effects are diarrhea and hand-foot syndrome. Capecitabine has been used with combination regimens with both oxaliplatin and irinotecan in metastatic colorectal cancer patients. Other oral fluoropyrimidines are tegafur-uracil, S1 (a prodrug of 5-FU), gimeracil and oteracil.

Irinotecan: Irinotecan is an anti-cancer drug by inhibition of topoisomerase I in metastatic colorectal cancer. It is active as monotherapy but combination with $\mathrm{FU}$ is more active than monotherapy. In several clinical studies showed that significant single-agent activity in colorectal cancer resistant to FU-based firstline therapy Firstly, clinical benefit of irinotecan has demonstrated after FU failure in patients with metastatic colorectal cancer with $15 \%$ response rates. ${ }^{12-14}$ Two first-line phase III trials ${ }^{15,16}$ showed a significant improvement in results with the addition of irinotecan to FU-LV combinations therapy (FOLFIRI) Following trials has demonstrated that the role of irinotecan in the first-line treatment. One year survival (36 versus 14 percent) and quality of life was superior with irinotecan combinations. ${ }^{13}$ Different schedules has similar therapeutic outcomes. Most using schedule is FOLFIRI regimen. The most common side effects of irinotecan are diarrhea, myelosuppression, and alopecia. 
Oxaliplatin: Oxaliplatin, a new cytotoxic agent from the diaminocyclohexane platinum family, has a mechanism of action similar to the other platinum derivates, with a different spectrum of antitumor activity against some tumor models; in particular, activity against colon cell lines and synergistic activity of oxaliplatin and FU in experimental models have been demonstrated. Oxaliplatin has limited activity as a single agent, but it shows enhanced efficacy of fluoropyrimidines. In three studies, combinations of oxaliplatin and FU/LV (FOLFOX) had higher antitumor activity compared to FU/ LV regimen. ${ }^{17-19}$ In first line therapy, a European randomized phase III trial ${ }^{17}$ demonstrated significant superiority of the combinations regimen of Oxaliplatin, LV, and bolus plus infusional FU (FOLFOX4) over the Mayo Clinic regimen in terms of response rate $(50 \% \mathrm{v} 22 \%$, respectively; P: 0.0001$)$ and progression-free survival $(8.2 \mathrm{\vee} 6.0$ months, respectively; p: 0.0003), with no statistical difference in median OS time (16.2 v 14.7 months, respectively; $p$ 0.12). The dose limiting toxicity of oxaliplatin is neurotoxicity. Nausea and vomiting, minimal myelotoxicity are other side effects.

Comparing irinotecan- and oxaliplatin: The outcomes of firstline oxaliplatin/FU/LV and irinotecan/FU/LV are similar in head to head comparisons. In the pivotal Intergroup N9741phase 3 trial demonstrated the superiority of FOLFOX compared with IFL as first-line therapy for colorectal cancer. Response rate (45\% vs. $31 \%$, $\mathrm{p}=0.002)$, PFS (8.7 months vs. 6.9 months, $\mathrm{p}=0.0014)$, and overall survival (19.5 months vs. 15.0 months, $\mathrm{p}=0.0001)$ were superior with oxaliplatin regimens in this trial. ${ }^{20}$ However, in two European trials and a Japanese trial, FOLFOX and FOLFIRI had similar efficacy. ${ }^{21-23}$ Combinations of 5-FU/LV, irinotecan, and oxaliplatin (FOLFOXIRI) show high activity but also increased toxicity. ${ }^{24}$

\section{Inhibitors of the VEGF system}

Angiogenesis is one of the essential causes of tumor progression. The VEGF/VEGF receptor pathway, in particular, contributes to several processes in tumor angiogenesis. and inhibition of VEGF is important in metastatic colorectal cancer treatment. The agents targeting VEGF are bevacizumab, aflibercept and ramucirumab. Bevacisumab improves outcomes in first line and next step of treatment. But aflibercept and ramucirumab can use after first line treatment in metastatic colorectal cancer.

Bevacizumab: Bevacizumab is a humanized monoclonal antibody targeting VEGF and it has demonstrated clinical efficacy for the treatment of metastatic colorectal cancer in combination with fluorouracil and LV alone or with irinotecan/oxaliplatin. ${ }^{25-28}$ But bevacizumab do not have significant single-agent activity in metastatic colorectal cancer. ${ }^{25}$ In a randomised trial addition of bevacizumab was associated with a significant 19 percent reduction in the risk of death, but this translated into a median overall survival advantage of only two months (19.8 versus 17.6 months) and median progression free survival was two months (9.1 versus 6.9 months). ${ }^{29}$ The comparison of IFL with or without bevacizumab was made in a trial of of 813 patients. The outcomes of this trial showed the benefit of addition bevacizumab in objective response rate ( 45 versus 35 percent), time to tumor progression (11 versus 6 months) and median survival (20 versus 16 months). ${ }^{30}$ The benefit of adding bevacizumab to a firstline oxaliplatin based regimens is demostrated in the TREE-2, ECOG 3200 and NO 16966 trials. ${ }^{31-33}$ On the other hand, in the phase III ITACa randomized trial,there was no benefit for PFS and overall survival for the addition of bevacizumab to FOLFOX4..$^{34}$ Bevacizumab also adds benefit to first line FU/LV and capecitabine. ${ }^{35,36}$ Bevacizumab has serious and potentially fatal adverse effects such as proteinuria, hypertension, bleeding, GI tract perforation, arterial and possibly venous thromboembolic events. ${ }^{37}$ Other rare side effects include reversible posterior leukoencephalopathy, nasal septum perforation, and jaw osteonecrosis.

Aflibercept: Intravenous aflibercept is a recombinant fusion protein consisting of human VEGF receptors 1 and 2. The FDA approved aflibercept in 2012 as a component of second-line therapy in combination with FOLFIRI. VELOUR trial showed that aflibercept was benefit for second line treatment after progression of oxaliplatin and bevecizumab based regimen and aflibercept had used with FOLFIRI in this trial. Median overall survival was significantly longer in patients treated with aflibercept (13.5 versus 12.1 months) and median PFS was longer (6.9 versus 4.7 months). ${ }^{38}$ The side effects of aflibercept is sismilar wtih bevacizumab.

Ramucirumab: Ramucirumab is a recombinant monoclonal antibody that binds to the VEGFR2. The FDA approved ramucirumab in 2015 as a component of second-line therapy in combination with FOLFIRI. The efficacy of ramucirumab for second line treatment of mCRC was studied in the phase III RAISE trial. In this trial the addition of ramucirumab to FOLFIRI as second line treatment for patients pretreated with a FOLFOX and bevacizumab improved overall survival (median overall survival 13.3 vs. 11.7 months; $p=0.022$ ) and PFS (5.7 versus 4.5 months). ${ }^{39}$ Grade 3 or worse side effects with ramucirumab included neutropenia ( 38 versus 23 percent), hypertension (11 versus 3 percent), and fatigue in this trial.

\section{Agents targetıng the EGFR}

The Epidermal Growth Factor Receptor (EGFR) is a transmembrane glycoprotein receptor. EGFR may be involved in autocrine or paracrine control of colorectal cancer (CRC) cell growth, or in the development of angiogenesis or metastases. ${ }^{40,41}$ Two monoclonal antibodies (MoAbs) targeting the EGFR are active for treatment of metastatic CRC (mCRC), cetuximab and panitumumab. Cetuximab is a chimeric IgG1 monoclonal antibody that binds to the extracellular domain of the EGFR. Panitumumab is a fully human IgG2 monoclonal antibody that also targets the EGFR. They have single-agent efficacy in advanced colorectal cancer and both cetuximab and panitumumab are only effective in the subset of patients whose tumors have wild type (WT) and not mutated RAS (NRAS, KRAS) oncogenes. Also Anti-EGFR monoclonal antibodies are not beneficial with BRAF mutated patients.

Cetuximab: The single-agent response rate of cetuximab is $10 \%{ }^{42}$ Cetuximab is useful in combination with irinotecan for patients with WT RAS tumors. The EPIC trial among oxaliplatin refractory patients showed that addition of cetuximab to irinotecan improved outcomes. ${ }^{43}$ In this trial, PFS was significantly higher with combined therapy (median 4 versus 2.6 months) and objective response rates (16 versus 4 percent). But median survival was not significantly different with combination therapy (10.7 versus 10 months). The BOND trial compared irinotecan plus weekly cetuximab versus cetuximab alone in 329 patients with irinotecan refractory mCRC..$^{44}$ Combined therapy was associated with a significantly better response rate $(23$ versus 11 percent) and TTP (4.1 versus 1.5 months). But there was no significantly difference about median survival ( 8.6 versus 6.9 months). The first line setting of cetuximab was evaluated in the CRYSTAL trial. 1198 patients with previously untreated $\mathrm{mCRC}$ were randomly assigned to FOLFIRI with or without cetuximab..$^{45}$ Median PFS was significantly better with cetuximab ( 8.9 versus 8 months) and overall response rate was better with cetuximab (47 versus 39 percent). But there was no significant overall survival benefit with cetuximab. 
However, among patients with WT KRAS; response rates, median PFS and overall survival were significantly higher with cetuximab arm. ${ }^{46}$ The OPUS trial compared weekly cetuximab plus FOLFOX4 versus FOLFOX 4 alone. ${ }^{47}$ Cetuximab with oxaliplatin was associated with a significantly higher response rate (57 versus 34 percent) but there was no significant overall survival benefit with combinations therapy. Both OPUS and CRYSTAL trials, patients receiving cetuximab had significantly higher rates of surgery for metastases and higher rates of complete (R0) resection. The COIN and NORDIC VII trials showed that no benefit for adding cetuximab to a first line Oxaliplatin regimen in patients with KRAS wildtype tumors. ${ }^{48,49}$

Panitumumab: The single-agent response rate of panitumumab was 10 percent in a multicenter trial. ${ }^{50}$ Panitumumab has efficacy of first, second, and thirdline panitumumab in combination with oxaliplatin or irinotecan based regimens in patients with WT RAS tumors. ${ }^{51,52}$ In the phase III PRIME trial, There was progression free survival benefit for FOLFOX plus panitumumab regimen (median PFS 9.6 versus 8 months) ${ }^{53}$ In the US by FDA panitumumab is indicated as a firstline therapy in combination with FOLFOX, but not with irinotecan containing regimens. The main toxicities of anti-EGFR antibodies are skin rash, hypomagnesemia, diarrhea, and hypersensitivity reactions, which is particularly relevant for the chimeric antibody cetuximab. ${ }^{54}$ The risk of infusion reactions with panitumumab is lower than with cetuximab.

\section{Comparison of EGFR monoclonal antibodies and bevacizumab}

EGFR antibodies and bevacizumab should not be combined in firstline therapy. EGFR targeted therapies and bevacizumab compared directly with three trials. These trials are FIRE, PEAK and CALGB/ South-west Oncology Group (SWOG) 80405.

The FIRE-3 trial compared the FOLFIRI plus cetuximab or FOLFIRI plus bevacizumab in 592 patients with KRAS exon 2 wild-type colorectal cancer. ${ }^{55}$ Median progression free survival (PFS) was similar (10.0 versus 10.3 months) and median overall survival was significantly longer with cetuximab (28.7 versus 25 months) patients The patients who had WT RAS status in KRAS exons 2, 3 and 4 and NRAS exons 2 and 3 had an even more pronounced survival benefit from cetuximab (median overall survival 33.1 versus 25.9 months). The PEAK trial compared the FOLFOX plus panitumumab or bevacizumab (56); In the patients with exon 2 WT KRAS, PFS was similar, but median overall survival was significantly better (34 versus 24 months). When the analysis was expanded to include patients who were WT in exons 2, 3, and 4 of KRAS and NRAS, panitumumab was associated with a significant improvement in PFS (median 41 versus 29 months).

In the CALGB/SWOG 80405 trial patients were randomly assigned to receiving cetuximab or bevacizumab with either FOLFOX or FOLFIRI. ${ }^{57}$ In a preliminary report at the 2014 ASCO annual meeting,

Table I Summary of the treatment of metastatic colorectal patients overall survival from was similar (29.9 months for cetuximab and 29 months for bevacizumab) and median PFS was similar (10.4 versus 10.8 months). After expanded RAS analysis, objective response rates were significantly higher with cetuximab (69 versus 54 percent), median overall survival was similar ( 32 months for cetuximab and 31.2 months for bevacizumab) and median PFS was similar. There were no benefit the dual antibody therapy (EGFR targeted therapies and bevacizumab together) in the BOND-2, PACCE and CAIRO-2 trials. ${ }^{8-60}$

\section{Patients with refractory disease}

Regorafenib: Regorafenib is a small molecule inhibitor of VEGF receptors 1 to 3 and oncogenic receptor tyrosine kinases. It has has efficacy in a salvage therapy in metastatic colorectal cancer. Median overall survival was higher for patients receiving regorafenib compared with placebo (6.4 vs. 5.0 months) in the phase 3 CORRECT trial. ${ }^{61}$ Regorafenib was also had an improvement of PFS (1.9 vs. 1.7 months). The most common severe toxicities observed with regorafenib were hand-foot skin reaction, fatigue, diarrhea, and hypertension. Regorafenib is FDA approved as a salvage therapy option in patients with advanced colorectal cancer who have previously been treated with a fluoropyrimidine, oxaliplatin, irinotecan, a VEGF inhibitor and, if KRAS wild-type, an EGFR monoclonal antibody.

Trifluridin-etipiracil (TAS-102): TAS-102 is an oral cytotoxic agent that consists the nucleoside analog trifluridine and tipiracil, a potent thymidine phosphorylase inhibitör. In the phase II trial, TAS 102 had significantly prolonged median overall survival ( 9 versus 6.6 months). ${ }^{62}$ In the phase 3 RECOURSE trial, TAS 102 was associated with a significant prolongation in median overall survival (7.1 versus 5.3 months). ${ }^{63}$ The most frequently toxicities were gastrointestinal and hematologic.

\section{Immunotherapeutic options}

\section{(PD-1/PD-L1 Immune checkpoint inhıbitors)}

Immune checkpoint inhibitors targeting the programmed death-1 (PD-1) pathway by binding to PD-1 or its ligand(s) (PD-L1/L2) have shown proof of efficacy in various malignancies since $2014 .{ }^{64}$ The importance of the immune system in the biology of CRC is underscored by the finding that infiltration of the tumor by specific $\mathrm{T}$ cell immune infiltrates is highly correlated with better disease free and overall survival at all tumor stages. MSI-H/dMMR colon cancers are commonly characterized by dense lymphocytic infiltrates indicating a potential activation of the host's immune system. ${ }^{65}$ In the pivotal pilot study was investigated the role of pembrolizumab in patients with advanced colorectal cancer with cohorts identified by their MSI status (MSI-H/dMMR vs. MSS/pMMR). ${ }^{66}$ Single-agent pembrolizumab showed an activity only among patients with MSI-H/dMMR cancers. There was a more than $60 \%$ response rate and a more than $90 \%$ disease control rate with some patients experiencing durable response for more than a year (Table 1).

\section{Metastatic Colorectal Cancer with Unresectable metastases}

Molecular testing

Any RAS mut (\%55) $\quad$ All RAS wt (\% 40)

CT doublet/triplet

\author{
CT doublet/triplet
}

+bevacisumab

First line $\quad$ CT doublet/tri
+ +bevacisumab

BRAF mut

CT doublet+EGFR inhibitor 
Table Continued....

\begin{tabular}{|c|c|c|c|c|c|c|c|}
\hline \multicolumn{8}{|c|}{ Metastatic Colorectal Cancer with Unresectable metastases } \\
\hline \multirow[t]{2}{*}{ Second line } & $\begin{array}{l}\text { CT doublet+VEGF } \\
\text { inhibitor }\end{array}$ & & $\begin{array}{l}\text { CT doublet+VEGF } \\
\text { inhibitor }\end{array}$ & & CT doublet+ & & EGFR inhibitör? \\
\hline & & & & & Bevacisumab & & +Chemotherapy \\
\hline Third line & Regorofanibe & TAS- 102 & $\begin{array}{l}\text { EGFR } \\
\text { inhibitör+irinotecan }\end{array}$ & & Regorafanib & TAS- 102 & Regorafanib \\
\hline \multirow[t]{2}{*}{ Fourth line } & BSC & & Regorafanib & TAS- 102 & BSC & & BSC \\
\hline & & & BSC & & & & \\
\hline
\end{tabular}

\section{Conclusion}

Colorectal cancer is the one of the field we had advance on management for the past ten year. Median survivals have prolonged from 12 months to nearly 40 months during this period. We assume that by the passing time we will have much more knowledge about the cancer cell and pathophisiology and this will reflect positive effects on patient management and outcome.

\section{Acknowledgement}

None.

\section{Conflict of interest}

Authors declare that there is no conflict of interest.

\section{References}

1. Siegel RL, Miller KD, Jemal A. Cancer statistics, 2015. CA Cancer J Clin. 2015;65:5-29.

2. Siegel R, Desantis C, Virgo K, et al. Cancer treatment and survivorship statistics. CA Cancer J Clin. 2012;62(4):220-241.

3. Siegel R, Naishadham D, Jemal A. Cancer statistics. CA Cancer J Clin. 2012;62(1):10-29.

4. Grothey A, Marshall JL. Optimizing palliative treatment of metastatic colorectal cancer in the era of biologic therapy. Oncology (Williston Park). 2007;21:553-564.

5. Sobrero AF, Aschele C, Bertino JR. Fluorouracil in colorectal cancera tale of two drugs: implications for biochemical modulation. J Clin Oncol. 1997;15(1):368-381.

6. Thirion P, Michiels S, Pignon JP, et al. Modulation of fluorouracil by leucovorin in patients with advanced colorectal cancer: an updated metaanalysis. J Clin Oncol. 2004;22:3766.

7. Buyse $\mathrm{M}$, Thirion $\mathrm{P}$, Carlson RW, et al. Relation between tumour response to firstline chemotherapy and survival in advanced colorectal cancer: a metaanalysis. MetaAnalysis Group in Cancer. Lancet. 2000; 356(9227):373.

8. Poon MA, O'Connell MJ, Moertel CG, et al. Biochemical modulation of fluorouracil: evidence of significant improvement of survival and quality of life in patients with advanced colorectal carcinoma. J Clin Oncol. 1989;7(10):1407-1418.

9. Petrelli N, Douglass HO Jr, Herrera L, et al. The modulation of fluorouracil with leucovorin in metastatic colorectal carcinoma: a prospective randomized phase III trial. Gastrointestinal Tumor Study Group. J Clin Oncol. 1989;7(10):1419-1426.

10. Cassidy J, Clarke S, Diaz-Rubio E, et al. XELOX vs. FOLFOX-4 as firstline therapy for metastatic colorectal cancer: NO16966 updated results. Br J Cancer. 2011;105(1):58-64.

11. Miwa M, Ura M, Nishida M, et al. Design of a novel oral fluoropyrimidine carbamate, capecitabine, which generates 5- fluorouracil selectively in tumours by enzymes concentrated in human liver and cancer tissue. Eur $J$ Cancer. 1998;34(8):1274-1281.

12. Rougier P, Van Cutsem E, Bajetta E, et al. Randomised trial of irinotecan versus fluorouracil by continuous infusion after fluorouracil failure in patients with metastatic colorectal cancer. Lancet. 1998;352(9138):1407.

13. Cunningham D, Pyrhönen S, James RD, et al. Randomised trial of irinotecan plus supportive care versus supportive care alone after fluorouracil failure for patients with metastatic colorectal cancer. Lancet. 1998;352(8):1413.

14. Kim GP, Sargent DJ, Mahoney MR, et al. Phase III noninferiority trial comparing irinotecan with oxaliplatin, fluorouracil, and leucovorin in patients with advanced colorectal carcinoma previously treated with fluorouracil: N9841. J Clin Oncol. 2009;27(17):2848.

15. Douillard JY, Cunningham D, Roth AD, et al. Irinotecan combined with fluorouracil compared with fluorouracil alone as first-line treatment for metastatic colorectal cancer: A multicentre randomised trial. Lancet. 355(9290):1041-1047.

16. Saltz LB, Cox JV, Blanke C, et al. Irinotecan plus fluorouracil and leucovorin for metastatic colorectal cancer: Irinotecan Study Group. $N$ Engl J Med. 343(13):905-914.

17. de Gramont A, Figer A, Seymour M, et al. Leucovorin and fluorouracil with or without oxaliplatin as first-line treatment in advanced colorectal cancer. J Clin Oncol. 2000;18(16):2938-2947.

18. Giacchetti S, Perpoint B, Zidani R, et al. Phase III multicenter randomized trial of oxaliplatin added to chronomodulated fluorouracil-leucovorin as first-line treatment of metastatic colorectal cancer. J Clin Oncol. 2000;18(1):136-147.

19. Grothey A, Deschler B, Kroening H, et al. Phase III study of bolus 5-fluorouracil (5-FU)/folinic acid (FA) (Mayo) vs. weekly high-dose $24 \mathrm{~h}$ 5 -FU infusion/FA + oxaliplatin (OXA) in advanced colorectal cancer (ACRC). Proc Am Soc Clin Oncol. 2002;21:129.

20. Goldberg RM, Sargent DJ, Morton RF, et al. A randomized controlled trial of fluorouracil plus leucovorin, irinotecan, and oxaliplatin combinations in patients with previously untreated metastatic colorectal cancer. J Clin Oncol. 2004;22(1):23-30.

21. Tournigand C, André T, Achille E, et al. FOLFIRI followed by FOLFOX6 or the reverse sequence in advanced colorectal cancer: a randomized GERCOR study. J Clin Oncol. 2004; 22(2):229-237.

22. Colucci G, Gebbia V, Paoletti G, et al. Phase III randomized trial of FOLFIRI versus FOLFOX4 in the treatment of advanced colorectal cancer: a multicenter study of the Gruppo Oncologico Dell'Italia Meridionale. J Clin Oncol. 2005;23(22):4866-4875.

23. Yamakazi K, Nagase M, Tamagawa H, et al. A randomized phase III trial of mFOLFOX6 plus bevacizumab versus FOLFIRI plus bevacizumab as firstline treatment for metastatic colorectal cancer: West Japan Oncology Group study 4407G (WJOG4407G) (abstract). J Clin Oncol. 2014;32.

24. Falcone A, Ricci S, Brunetti I, et al. Phase III trial of infusional fluorouracil, leucovorin, oxaliplatin, and irinotecan (FOLFOXIRI) compared with infusional fluorouracil, leucovorin, and irinotecan (FOLFIRI) as first-line 
treatment for metastatic colorectal cancer: the Gruppo Oncologico Nor Ovest. J Clin Oncol. 2007;25(13):1670-1676

25. Giantonio BJ, Catalano PJ, Meropol NJ, et al. Bevacizumab in combination with oxaliplatin, fluorouracil, and leucovorin (FOLFOX4) for previously treated metastatic colorectal cancer: results from the Eastern Cooperative Oncology Group Study E3200. J Clin Oncol. 2007;25(12):1539-1544.

26. Kabbinavar FF, Hambleton J, Mass RD, et al. Combined analysis of efficacy: the addition of bevacizumab to fluorouracil/leucovorin improves survival for patients with metastatic colorectal cancer. J Clin Oncol. 2005;23(16):3706-3712.

27. Saltz LB, Clarke S, Diaz-Rubio E, et al. Bevacizumab in combination with oxaliplatin-based chemotherapy as first-line therapy in metastatic colorectal cancer: a randomized phase III study. J Clin Oncol. 2008;26(12):2013-2019.

28. Fuchs CS, Marshall J, Mitchell E, et al. Randomized, controlled trial of irinotecan plus infusional, bolus, or oral fluoropyrimidines in first-line treatment of metastatic colorectal cancer: results from the BICC-C Study. J Clin Oncol. 2007;25(30):4779-4786.

29. Hurwitz HI, Tebbutt NC, Kabbinavar F, et al. Efficacy and safety of bevacizumab in metastatic colorectal cancer: pooled analysis from seven randomized controlled trials. Oncologist. 2013;18(9):1004-1012.

30. Hurwitz H, Fehrenbacher L, Novotny W, et al. Bevacizumab plus irinotecan, fluorouracil, and leucovorin for metastatic colorectal cancer N Engl J Med. 2004;350:2335.

31. Hochster HS, Hart LL, Ramanathan RK, et al. Safety and efficacy of oxaliplatin and fluoropyrimidine regimens with or without bevacizumab as firstline treatment of metastatic colorectal cancer: results of the TREE Study. J Clin Oncol. 2008;26(21):3523-3529.

32. Giantonio BJ, Catalano PJ, Meropol NJ, et al. Bevacizumab in combination with oxaliplatin, fluorouracil, and leucovorin (FOLFOX4) for previously treated metastatic colorectal cancer: results from the Eastern Cooperative Oncology Group Study E3200. J Clin Oncol 2007; 25:1539.

33. Saltz LB, Clarke S, DíazRubio E, et al. Bevacizumab in combination with oxaliplatinbased chemotherapy as firstline therapy in metastatic colorecta cancer: a randomized phase III study. J Clin Oncol 2008; 26:2013.

34. Passardi A, Nanni O, Tassinari D, et al. Effectiveness of bevacizumab added to standard chemotherapy in metastatic colorectal cancer: final results for firstline treatment from the ITACa randomized clinical trial. Ann Oncol. 2015;26(6):1201-1207.

35. Vincenzi B, Santini D, Russo A, et al. Bevacizumab in association with de Gramont 5fluorouracil/ folinic acid in patients with oxaliplatin, irinotecan, and cetuximabrefractory colorectal cancer: a singlecenter phase 2 trial. Cancer. 2009;115(20):4849-4856

36. Tebbutt NC, Wilson K, Gebski VJ, et al. Capecitabine, bevacizumab, and mitomycin in firstline treatment of metastatic colorectal cancer: results of the Australasian Gastrointestinal Trials Group Randomized Phase III MAX Study. J Clin Oncol. 2010;28(19):3191-3198.

37. Ranpura V, Hapani S, Wu S. Treatmentrelated mortality with bevacizumab in cancer patients: a metaanalysis. JAMA. 2011; 305(5):487-494.

38. Van Cutsem E, Tabernero J, Lakomy R, et al. Addition of aflibercept to fluorouracil, leucovorin, and irinotecan improves survival in a phase III randomized trial in patients with metastatic colorectal cancer previously treated with an oxaliplatinbased regimen. JClin Oncol. 2012;30(28):34993506.

39. Tabernero J, Yoshino T, Cohn AL, et al. Ramucirumab versus placebo in combination with second-line FOLFIRI in patients with metastatic colorectal carcinoma that progressed during or after first-line therapy with bevacizumab, oxaliplatin, and a fluoropyrimidine (RAISE): a randomised, double-blind, multicentre, phase 3 study. Lancet Oncol. 2015;16(5):499-508
40. Kawamoto K, Onodera H, Kan S, et al. Possible paracrine mechanism of insulinlike growth factor 2 in the development of liver metastases from colorectal carcinoma. Cancer. 1999;85(1):18-25.

41. elHariry I, Pignatelli M, Lemoine N. Fibroblast growth factor 1 and fibroblast growth factor 2 immunoreactivity in gastrointestinal tumours. $J$ Pathol. 1997;181(1):39-45.

42. Chen HX, Mooney M, Boron M, et al. Phase II multicenter trial of bevacizumab plus fluorouracil and leucovorin in patients with advanced refractory colorectal cancer: an NCI Treatment Referral Center Trial TRC0301. J Clin Oncol. 2006; 24(21):3354-3360.

43. Sobrero AF, Maurel J, Fehrenbacher L, et al. EPIC: phase III tria of cetuximab plus irinotecan after fluoropyrimidine and oxaliplatin failure in patients with metastatic colorectal cancer. J Clin Oncol. 2008;26(14):2311-2319.

44. Cunningham D, Humblet Y, Siena S, et al. Cetuximab monotherapy and cetuximab plus irinotecan in irinotecanrefractory metastatic colorectal cancer. N Engl J Med. 2004;351:337-345.

45. Van Cutsem E, Köhne CH, Hitre E, et al. Cetuximab and chemotherapy as initial treatment for metastatic colorectal cancer. $N \mathrm{Engl} \mathrm{J} \mathrm{Med}$. 2009;360(14):1408-1417

46. Van Cutsem E, Köhne CH, Láng I, et al. Cetuximab plus irinotecan, fluorouracil, and leucovorin as firstline treatment for metastatic colorecta cancer: updated analysis of overall survival according to tumor KRAS and BRAF mutation status. J Clin Oncol. 2011; 29(15):2011-2019.

47. Bokemeyer C, Bondarenko I, Hartmann JT, et al. Efficacy according to biomarker status of cetuximab plus FOLFOX4 as firstline treatment for metastatic colorectal cancer: the OPUS study. Ann Oncol. 2011;22(7):1535-1546

48. Maughan TS, Adams RA, Smith CG, et al. Addition of cetuximab to oxaliplatinbased firstline combination chemotherapy for treatment of advanced colorectal cancer: results of the randomised phase $3 \mathrm{MRC}$ COIN trial. Lancet. 2011;377(9783):2103-2114

49. Tveit KM, Guren T, Glimelius B, et al. Phase III trial of cetuximab with continuous or intermittent fluorouracil, leucovorin, and oxaliplatin (Nordic FLOX) versus FLOX alone in firstline treatment of metastatic colorectal cancer: the NORDICVII study. J Clin Oncol. 2012;30(15):1755-1762.

50. Van Cutsem E, Peeters M, Siena S, et al. Openlabel phase III trial of panitumumab plus best supportive care compared with best supportive care alone in patients with chemotherapyrefractory metastatic colorectal cancer. J Clin Oncol. 2007;25(13):1658-1662.

51. Douillard JY, Siena S, Cassidy J, et al. Randomized, phase III trial of panitumumab with infusional fluorouracil, leucovorin, and oxaliplatin (FOLFOX4) versus FOLFOX4 alone as firstline treatment in patients with previously untreated metastatic colorectal cancer: the PRIME study. $J$ Clin Oncol. 2010;28(31):4697-4705.

52. Peeters M, Price TJ, Cervantes A, et al. Final results from a randomized phase 3 study of FOLFIRI $\{+/\}$ panitumumab for secondline treatment of metastatic colorectal cancer. Ann Oncol. 2014;25(1):107-116.

53. Douillard JY, Siena S, Cassidy J, et al. Randomized, phase III trial of panitumumab with infusional fluorouracil, leucovorin, and oxaliplatin (FOLFOX4) versus FOLFOX4 alone as firstline treatment in patients with previously untreated metastatic colorectal cancer: the PRIME study. J Clin Oncol 2010; 28:4697.

54. Grothey A. Recognizing and managing toxicities of molecular targeted therapies for colorectal cancer. Oncology (Williston Park). 2006;20:2128

55. Heinemann V, von Weikersthal LF, Decker T, et al. FOLFIRI plus cetuximab versus FOLFIRI plus bevacizumab as first-line treatment for patients with metastatic colorectal cancer (FIRE-3): a randomised, openlabel, phase 3 trial. Lancet Oncol. 2014;15:1065-1075. 
56. Schwartzberg LS, Rivera F, Karthaus M, et al. PEAK: a randomized, multicenter phase II study of panitumumab plus modified fluorouracil, leucovorin, and oxaliplatin (mFOLFOX6) or bevacizumab plus mFOLFOX6 in patients with previously untreated, unresectable, wildtype KRAS exon 2 metastatic colorectal cancer. J Clin Oncol. 2014;32(21):2240-2247.

57. Venook AP, Niedzwiecki D, Lenz HJ, et al. CALGB/SWOG 80405: Phase III trial of irinotecan/5FU/ leucovorin (FOLFIRI) or oxaliplatin/5FU/ leucovorin (mFOLFOX6) with bevacizumab (BV) or cetuximab (CET) for patients (pts) with KRAS wildtype (wt) untreated metastatic adenocarcinoma of the colon or rectum (MCRC) (abstract). J Clin Oncol. $2014 ; 32$.

58. Saltz LB, Lenz HJ, Kindler HL, et al. Randomized phase II trial of cetuximab, bevacizumab, and irinotecan compared with cetuximab and bevacizumab alone in irinotecanrefractory colorectal cancer: the BOND2 study. J Clin Oncol. 2007;25(29):4557-4561.

59. Hecht JR, Mitchell E, Chidiac T, et al. A randomized phase IIIB tria of chemotherapy, bevacizumab, and panitumumab compared with chemotherapy and bevacizumab alone for metastatic colorectal cancer. $J$ Clin Oncol. 2009;27(5):672-680.

60. Tol J, Koopman M, Cats A, et al. Chemotherapy, bevacizumab, and cetuximab in metastatic colorectal cancer. NEngl JMed. 2009;360(6):563572 .

61. Grothey A, Van Cutsem E, Sobrero A, et al. Regorafenib monotherapy for previously treated metastatic colorectal cancer (CORRECT): an international, multicentre, randomised, placebo-controlled, phase 3 trial. Lancet. 2013;381(9863):303-332.

62. Yoshino T, Mizunuma N, Yamazaki K, et al. TAS102 monotherapy for pretreated metastatic colorectal cancer: a doubleblind, randomised, placebocontrolled phase 2 trial. Lancet Oncol. 2012;13(10):993-1001.

63. Mayer RJ, Van Cutsem E, Falcone A, et al. Randomized trial of TAS102 for refractory metastatic colorectal cancer. N Engl J Med. 2015;372:1909.

64. Koster BD, de Gruij1 TD, van den Eertwegh AJ. Recent developments and future challenges in immune checkpoint inhibitory cancer treatment. Curr Opin Oncol. 2015;27(6):482-488.

65. Smyrk TC, Watson P, Kaul K, et al. Tumor-infiltrating lymphocytes are a marker for microsatellite instability in colorectal carcinoma. Cancer. 2001;91(12):2417-2422.

66. Le DT, Uram JN, Wang H, et al. PD-1 Blockade in tumors with mismatchrepair deficiency. $N$ Engl J Med. 2015;372:2509-2520. 